\title{
Neimark-Sacker Bifurcation in Demand-Inventory Model with Stock-Level-Dependent Demand
}

\author{
Piotr Hachuła, ${ }^{1}$ Magdalena Nockowska-Rosiak, ${ }^{2}$ and Ewa Schmeidel ${ }^{3}$ \\ ${ }^{1}$ Institute of Logistics and Warehousing, Poznań, Poland \\ ${ }^{2}$ Lodz University of Technology, Łódź, Poland \\ ${ }^{3}$ University of Białystok, Bialystok, Poland \\ Correspondence should be addressed to Ewa Schmeidel; eschmeidel@math.uwb.edu.pl
}

Received 4 September 2016; Accepted 20 November 2016; Published 1 January 2017

Academic Editor: Zhan Zhou

Copyright (C) 2017 Piotr Hachuła et al. This is an open access article distributed under the Creative Commons Attribution License, which permits unrestricted use, distribution, and reproduction in any medium, provided the original work is properly cited.

\begin{abstract}
An analysis of dynamics of demand-inventory model with stock-level-dependent demand formulated as a three-dimensional system of difference equations with four parameters is considered. By reducing the model to the planar system with five parameters, an analysis of one-parameter bifurcation of equilibrium points is presented. By the analytical method, we prove that nondegeneracy conditions for the existence of Neimark-Sacker bifurcation for the planar system are fulfilled. To check the sign of the first Lyapunov coefficient of Neimark-Sacker bifurcation, we use numerical simulations. We give phase portraits of the planar system to confirm the previous analytical results and show new interesting complex dynamical behaviours emerging in it. Finally, the economical interpretation of the system is given.
\end{abstract}

\section{Introduction}

Great economic development after the second world war has released a need of development of mathematical methods to support optimization of economic and business processes. Material flow, production, and inventory are aspects of a business, which, to make it profitable, need to be optimized. Therefore, many models of supply chain were created in the mid-20th century. To mention the most noticeable, we ought to list works of Wagner and Whitin [1], Brown [2], and Holt et al. (HMMS model) [3]. They have laid the foundation for supply chain modelling. Those models, despite being relatively simple, have become an inspiration for contemporary researchers: to redefine models in order to fit to the current challenges and to analyse them using available computation power, for example, [4-6].

Prediction of future demand and inventory is an important aspect of running and managing manufacturing or trade company. Methods supporting those tasks have been developed by economists already in the mid-20th century; nonetheless, they are still being improved, as economy still changes and creates new challenges. One of those methods is modelling of economic phenomena using mathematical formulations. The topic of demand and inventory needs a contextual approach, since many factors can influence it and different views may be needed. Therefore, the models are created with the usage of different mathematical tools. We can mention here recent works related to demand and inventory that investigate and describe specific economic cases: Chen and $\mathrm{Hu}$ in [7] consider an inventory and pricing model and develop polynomial time algorithms to maximize the total profit; Mondal et al. in [8] consider generic algorithm for the case of inventory of a deteriorating item; Qi et al. in [4] consider supply chain that experiences a disruption in demand during the planning horizon.

Ma and Feng in [6] proposed the dynamical model of demand and inventory with mechanism of demand stimulation and inventory limitation. Nowakowska in [9] showed application of such a model to electrical energy market. Hachuła et al. analysed the stability of equilibrium points in $[10,11]$.

The model describes demand and inventory of a product at one echelon of supply chain at the retailer. The considered supply chain consists of three echelons: manufacturer, 
retailer, and customers. The following rules are applied to the model: customers buy a good from a retailer; a retailer orders a product in the forecasted amount and forecast is prepared using single exponential smoothing Brown model [12]; a manufacturer produces and delivers exactly the ordered amount and the production capacity is unlimited; customers' demand depends on a retail price, which can be changed by a discount; price cannot be arbitrarily changed but the retailer can offer a discount depending on stock volume.

The model takes a form of the following system of difference equations:

$$
\begin{aligned}
& D_{n+1}=\left[\frac{A T}{(A+1) T-S_{n}}\right]^{k} D_{n}, \\
& S_{n+1}=S_{n}-D_{n}+\check{D}_{n}, \\
& \check{D}_{n+1}=\alpha D_{n}+(1-\alpha) \check{D}_{n},
\end{aligned}
$$

where $n=0,1,2, \ldots$ indicates instance of time; $S_{n}$ is a stock volume at $n ; S_{n}$ is a real number; interpretation of nonnegative values is obvious and the negative value of stock informs what amount is missing to fulfill demand and needs to be overcompensated in the next periods; $D_{n}$ is a demand volume at $n$, which assumes constant demand elasticity (see [13, p. 280]), $D_{n} \geq 0 ; \check{D}_{n}$ is a forecast of demand at $n$ and order placed by a retailer at a manufacturer; moreover, by assumption of unlimited capacity, it is also the delivered quantity at $n, \check{D}_{n} \geq 0$; $A$ is a parameter for discount steering, $A>0$; $T$ is a parameter for defining the target stock, $T>0 ; k$ is a price elasticity coefficient that regulates dependence between price, discount, and demand, $k>0 ; \alpha$ is a forecast smoothing coefficient of Brown model, $\alpha \in(0,1)$.

The first equation describes the influence of relation between current stock $S_{n}$ and target stock $T$ on future demand $D_{n+1}$, in a way that when stock is high, the retailer offers a discount to encourage customers to buy a product. The second equation lets us calculate future stock $S_{n+1}$ as a sum of current stock $S_{n}$ and delivery of amount of forecasted demand $\check{D}_{n}$ diminished by actual sales $D_{n}$. The third equation describes a method of demand forecasting. Future demand is forecasted using single exponential smoothing by Brown [2]. Smoothing coefficient $\alpha$ split importance between actual demand (sales) $D_{n}$ and forecasted demand $D_{n}$.

We analyse properties of a given model (1) on the ground of theory of difference equations. Three-dimensional difference systems were studied by many authors, for example, in [14-17]. Under some assumptions, such systems can be rewritten as third-order difference equations. Asymptotic properties of these types of equations were investigated in [18-23]. For the background of difference equations theory, see monographs $[24,25]$. Firstly, we construct a positive invariant set of the three-dimensional system. We notice that the given system has an invariant plane and can be reduced to the family of the planar systems, which we construct positive invariant sets and provide the equilibrium points for. We recall the properties of equilibrium points obtained in [11]. The aim of this paper is to analyse the dynamics of planar systems on the ground of bifurcation theory (see [26]) and numerical simulations. It was shown that nondegeneracy conditions for Neimark-Sacker bifurcation are fulfilled and existence of transcritical bifurcation is suggested. The analytical results are confirmed by numerical simulations. Finally, the economical interpretation of the system is given.

\section{Main Results}

For the sake of convenience, let us write the model as a system of three first-order difference equations with $x, y$, and $z$ replacing $D, S$, and $\check{D}$, respectively, in system (1),

$$
\begin{aligned}
& x_{n+1}=\left[\frac{A T}{(A+1) T-y_{n}}\right]^{k} x_{n}, \\
& y_{n+1}=y_{n}-x_{n}+z_{n}, \\
& z_{n+1}=\alpha x_{n}+(1-\alpha) z_{n},
\end{aligned}
$$

with $x_{n}, z_{n} \geqslant 0, y_{n}<(A+1) T$, or as $\left(x_{n+1}, y_{n+1}, z_{n+1}\right)=$ $f\left(x_{n}, y_{n}, z_{n}\right)$ with a mapping:

$$
\begin{aligned}
f(x, y, z) \\
\quad=\left(\frac{(A T)^{k}}{((A+1) T-y)^{k}} x, y-x+z, \alpha x+(1-\alpha) z\right) .
\end{aligned}
$$

It is easy to see that $(0, y, 0)$ for $y<(A+1) T$ and $(x, T, x)$ for $x \geq 0$ are equilibrium points of the system $\left(x_{n+1}, y_{n+1}, z_{n+1}\right)=$ $f\left(x_{n}, y_{n}, z_{n}\right)$.

Let us shortly elaborate on the meaning of those equilibrium points. The goal of the retailer is to reach target stock $T$. If additionally actual demand $x_{n}$ and forecasted demand $z_{n}$ are equal and greater than zero, the retailer's stock level does not change in the next period, which means that system (2) is in the equilibrium point $(x, T, x)$. On the other hand, when $x_{n}=z_{n}=0$, the retailer's stock level $y$ cannot be changed. This means that the retailer remains in equilibrium point $(0, y, 0)$.

We recall that $B_{\mathrm{INV}}^{f}$ is the positive invariant set of the system $\left(x_{n+1}, y_{n+1}, z_{n+1}\right)=f\left(x_{n}, y_{n}, z_{n}\right)$ if $B_{\mathrm{INV}}^{f} \subset D_{f}$ and $f\left(B_{\mathrm{INV}}^{f}\right) \subset B_{\mathrm{INV}}^{f}$.

Proposition 1 (see [11]). Here,

$$
\begin{aligned}
& B_{\mathrm{INV}}^{f} \\
& =\left\{(x, y, z) \in \mathbb{R}^{3}: x, z \geq 0, y+\alpha^{-1} z<(A+1) T\right\} \\
& \quad \cup\{(x, T, x): x \geq \alpha A T\}
\end{aligned}
$$

is the positive invariant set of the system $\left(x_{n+1}, y_{n+1}, z_{n+1}\right)=$ $f\left(x_{n}, y_{n}, z_{n}\right)$, where $f$ is given by (3).

One can observe that the second and the third equation of system (2) are linear ones. Therefore, we treat them with some linear transformations in order to obtain a more convenient form for analysis: both sides of the second equation 
multiplied by $\alpha$ and the second and the third equation added. As an output, we get

$$
\alpha\left(y_{n+1}-y_{n}\right)=z_{n}-z_{n+1},
$$

which enables us to state the following.

Corollary 2. System (2) has the invariant plane $\alpha y+z=\alpha y_{1}+$ $z_{1}$ for any $y_{1} \in \mathbb{R}, z_{1} \geq 0, \alpha y_{1}+z_{1}<\alpha(A+1) T$. Hence, system (2) can be reduced to a family of planar systems:

$$
\begin{aligned}
& x_{n+1}=\left[\frac{A T}{(A+1) T-y_{n}}\right]^{k} x_{n}, \\
& y_{n+1}=(1-\alpha) y_{n}-x_{n}+\alpha s,
\end{aligned}
$$

with $s<(A+1) T$, where $s:=y_{1}+z_{1} / \alpha, y_{1} \in \mathbb{R}, z_{1} \geq 0$.

Proof. From (5), we get the first-order recursion of the form $\alpha y_{n+1}+z_{n+1}=\alpha y_{n}+z_{n}$ which implies that $\alpha y_{n}+z_{n}=\alpha y_{1}+z_{1}$. Constraint of the expression $\alpha y_{1}+z_{1}$ is a direct consequence of the positive invariant set construction.

Let us later write our systems (6) in the form of a family of mappings:

$$
\begin{aligned}
g_{s} & (x, y) \\
\quad & =\left(\left[\frac{A T}{(A+1) T-y}\right]^{k} x,(1-\alpha) y-x+\alpha s\right),
\end{aligned}
$$

for $s<(A+1) T$. Taking into consideration economic interpretation, $g_{s}: D_{g_{s}} \rightarrow \mathbb{R}^{2}$, where $D_{g_{s}}:=[0, \infty) \times$ $(-\infty,(A+1) T)$.

Proposition 3. Let $A, T, k>0, \alpha \in(0,1)$, and $s<(A+1) T$. The set $B_{\mathrm{INV}}^{g_{s}}=D_{g_{s}}$ is the positive invariant set of the system $\left(x_{n+1}, y_{n+1}\right)=g_{s}\left(x_{n}, y_{n}\right)$, where $g_{s}$ is given by $(7)$.

Proof. Let $s<(A+1) T$. For any $x \geq 0, y<(A+1) T$, we have $\left(g_{s}\right)_{1}(x, y) \geq 0$ and $\left(g_{s}\right)_{2}(x, y)=(1-\alpha) y-x+\alpha s<$ $(1-\alpha)(A+1) T+\alpha(A+1) T=(A+1) T$.

Mapping (7) may have at most two equilibrium points:

(i) $(0, s)$

(ii) $(\alpha(s-T), T)$ iff $s>T$

Stability of the equilibrium points of (2) has been examined in [11]. Based on the findings of [11], one can formulate the conclusions about the existence and stability of equilibrium points of planar systems (6), which are presented in Table 1. Now, using these results, we analyse the dynamics of the planar systems using bifurcation theory. We are especially interested in proving the existence of Neimark-Sacker bifurcation.

Graphs illustrating the cases in Table 1 are presented in Figures 1-4.

The planar system is dependent on five parameters $\alpha$, $A, T, k$, and $s$. The most interesting case in the bifurcation analysis for the planar system (6) is dependence on parameter
TABLE 1: Overview of conditions for existence and stability of the equilibrium points of (6).

\begin{tabular}{cccc}
\hline & Conditions on parameters & $(0, s)$ & $(\alpha(s-T), T)$ \\
\hline C1 & $T>s$ & Stable & Does not exist \\
C2 & $T=s$ & Unstable & Does not exist \\
C3 & $T<s<(A+1) T, k \leq 1$ & Unstable & Stable \\
C4 & $T<s<(1+A / k) T, k>1$ & Unstable & Stable \\
C5 & $(1+A / k) T<s<(1+A) T, k>1$ & Unstable & Unstable \\
\hline
\end{tabular}

$s$ with fixed $A_{0}, T_{0}, k_{0}>0, \alpha_{0} \in(0,1)$, which now we will carry on.

From the conditions in Table 1, in order to examine bifurcations on parameter $s$, we have to consider two cases dependent on $k_{0}$.

Case $1\left(k_{0} \in(0,1]\right)$. For $s<T_{0}$, the equilibrium point $(0, s)$ is the only equilibrium point of the system $\left(x_{n+1}, y_{n+1}\right)=$ $g_{s}\left(x_{n}, y_{n}\right)$ which is stable. For $s>T_{0}$, the considered system possesses two equilibrium points: unstable $(0, s)$ and stable $\left(\alpha_{0}\left(s-T_{0}\right), T_{0}\right)$. This suggests that $s=T_{0}$ is the bifurcation parameter for the transcritical bifurcation.

Case $2\left(k_{0}>1\right)$. For $s=T_{0}$, probably the transcritical bifurcation occurs like in the previous case. For $s<(1+$ $\left.A_{0} / k_{0}\right) T_{0}$, the equilibrium point $\left(\alpha_{0}\left(s-T_{0}\right), T_{0}\right)$ is stable and loses its stability for $s>\left(1+A_{0} / k_{0}\right) T_{0}$. We prove that, for $s=\left(1+A_{0} / k_{0}\right) T_{0}$, the Neimark-Sacker bifurcation occurs. We use classical analytical results from [26] and numerical simulations. For the sake of convenience, let us quote from [26] Theorem 4.5 as Lemma 4 and Theorem 4.6 as Lemma 5.

Lemma 4 (Theorem 4.5, [26]). Suppose that a twodimensional discrete-time system $x \rightarrow f(x, \alpha), x \in \mathbb{R}^{2}, \alpha \in$ $\mathbb{R}^{1}$, with smooth $f$, has, for all sufficiently small $|\alpha|$, the fixed point $x=0$ with multipliers $\mu_{1,2}(\alpha)=r(\alpha) e^{ \pm i \phi(\alpha)}$, where $r(0)=$ $1, \phi(0)=\theta_{0}$. Let the following conditions be satisfied:

(C.1) $r^{\prime}(0) \neq 0$.

(C.2) $e^{i k \theta_{0}} \neq 1$ for $k=1,2,3,4$.

Then, there are smooth invertible coordinate and parameter changes transforming the system into

$$
\begin{aligned}
& \left(\begin{array}{l}
y_{1} \\
y_{2}
\end{array}\right) \longrightarrow(1+\beta)\left(\begin{array}{cc}
\cos \theta(\beta) & -\sin \theta(\beta) \\
\sin \theta(\beta) & \cos \theta(\beta)
\end{array}\right)\left(\begin{array}{l}
y_{1} \\
y_{2}
\end{array}\right) \\
& +\left(y_{1}^{2}+y_{2}^{2}\right) \\
& +\left(\begin{array}{cc}
\cos \theta(\beta) & -\sin \theta(\beta) \\
\sin \theta(\beta) & \cos \theta(\beta)
\end{array}\right)\left(\begin{array}{cc}
a(\beta) & -b(\beta) \\
b(\beta) & a(\beta)
\end{array}\right)\left(\begin{array}{l}
y_{1} \\
y_{2}
\end{array}\right) \\
& +O\left(\|y\|^{4}\right),
\end{aligned}
$$

with $\theta(0)=\theta_{0}$ and $a(0)=\operatorname{Re}\left(e^{-i \theta_{0}} c_{1}(0)\right)$, where $c_{1}(0)$ is given by formula (4.21) in [26]. 


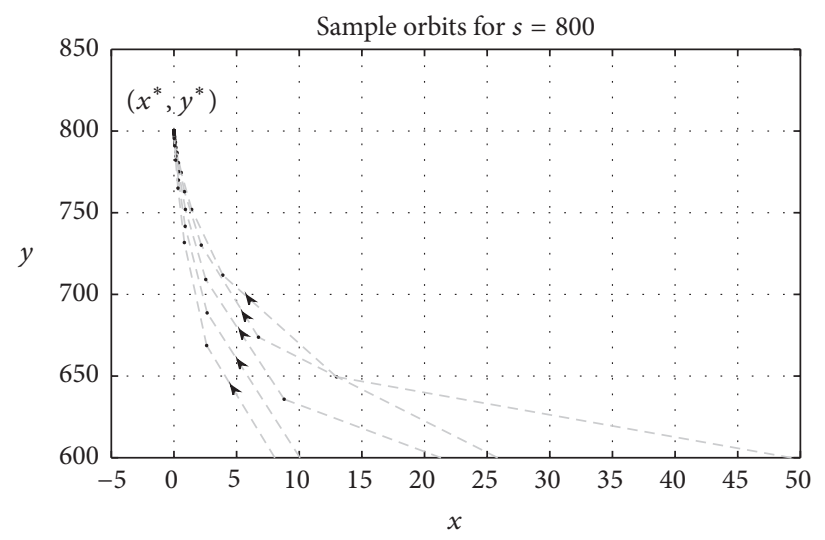

(C1)

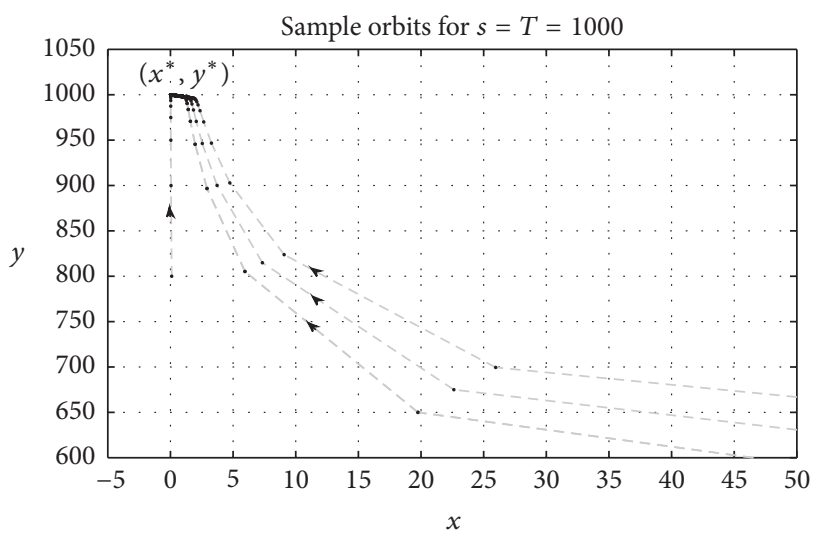

(C2a)

Figure 1: Examples illustrating conditions (C1) and (C2) from Table 1.

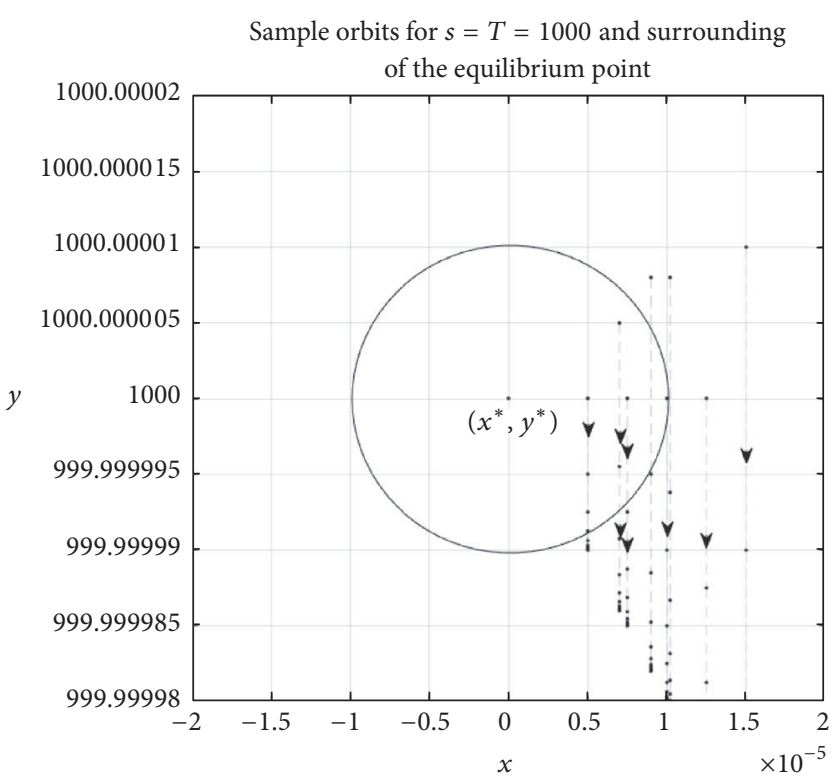

(C2b)

FIgure 2: Example illustrating condition (C2) from Table 1.

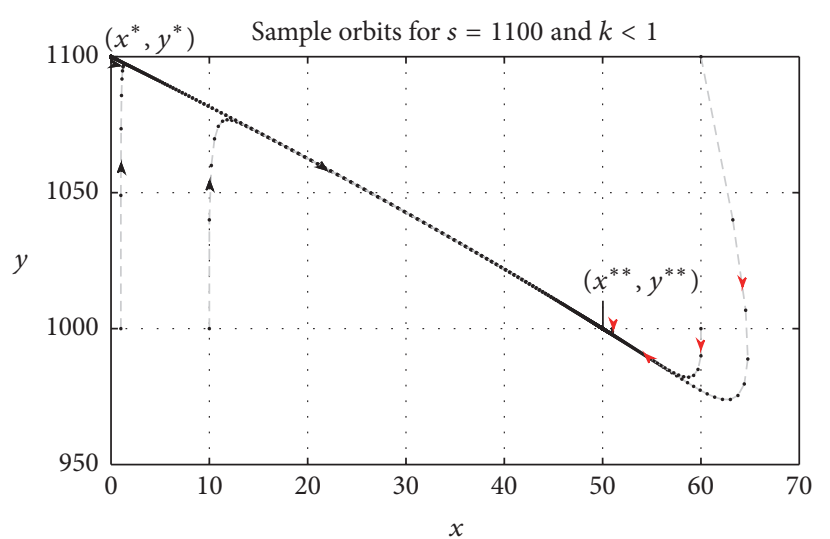

(C3)

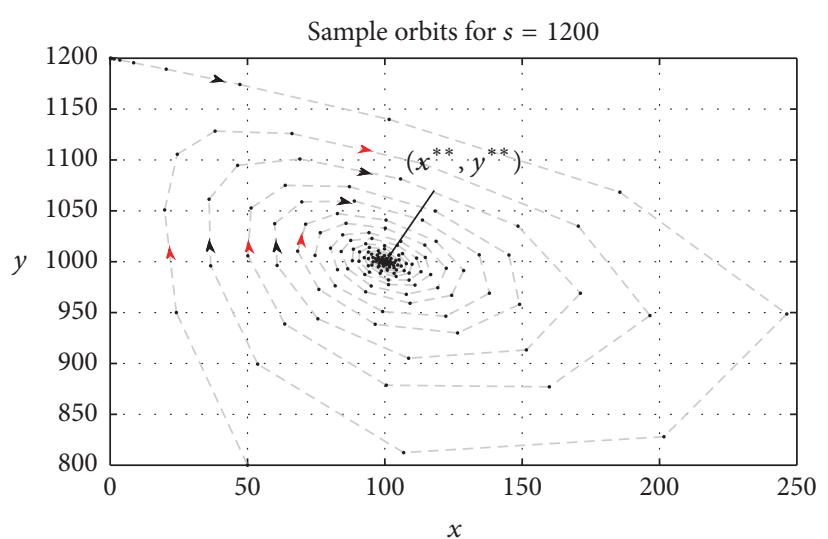

(C4)

Figure 3: Examples illustrating conditions (C3) and (C4) from Table 1. 


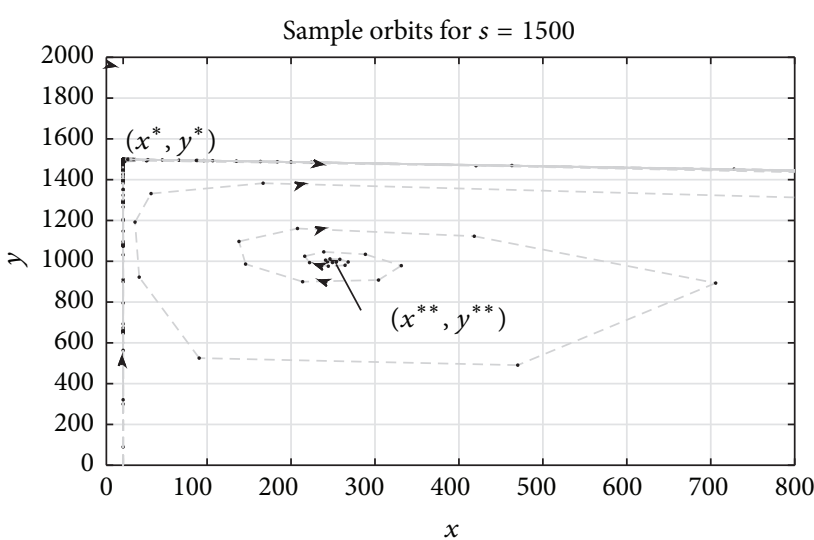

(C5)

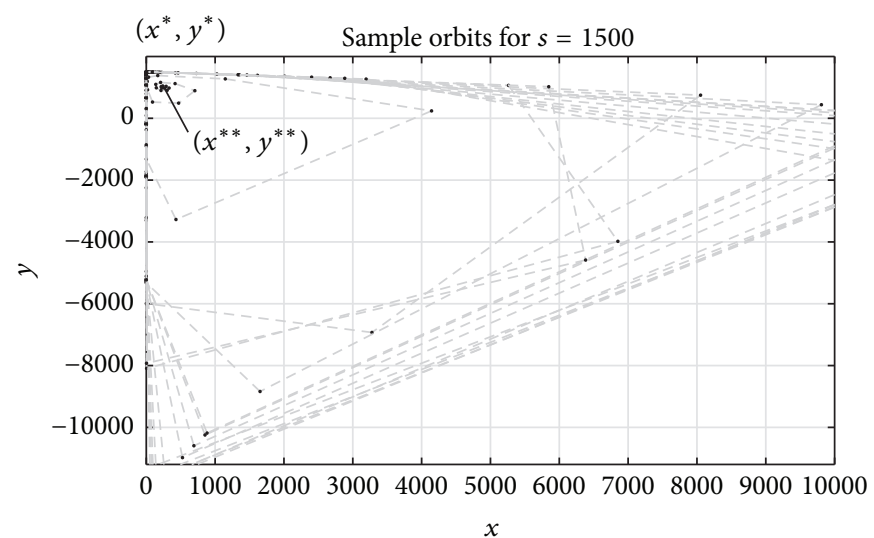

(C5)

Figure 4: Examples illustrating condition (C5) from Table 1. The right figure is a "big picture" of the same case.

Lemma 5 ((Theorem 4.6, [26]) (generic Neimark-Sacker bifurcation)). For any generic two-dimensional one-parameter system $x \rightarrow f(x, \alpha)$, having at $\alpha=0$ the fixed point $x_{0}=$ 0 with complex multipliers $\mu_{1,2}(\alpha)=e^{ \pm i \theta_{0}}$, there is a neighbourhood of $x_{0}$ in which a unique closed invariant curve bifurcates from $x_{0}$ as $\alpha$ passes through zero.

Remark 6 (see [26]). The genericity conditions assumed in the theorem are the transversality condition (C.1) and the nondegeneracy condition (C.2) from Lemma 4 and the additional nondegeneracy condition:

$$
\text { (C.3) } a(0) \neq 0 \text {. }
$$

We start with translation mapping $g_{s}$ to the mapping which possesses fixed point in $(0,0)$ for sufficiently small $s$. We consider the $C^{2}$ mapping $G_{s}: \mathbb{R} \times\left(-\infty, A_{0} T_{0}\right) \rightarrow \mathbb{R}^{2}$ with parameter $s \in \mathbb{R}$ :

$$
\begin{aligned}
& G_{s}(x, y):=\left(\frac{\left(A_{0} T_{0}\right)^{k_{0}}}{\left(A_{0} T_{0}-y\right)^{k_{0}}}\left(x+\alpha_{0}\left(s+\frac{A_{0} T_{0}}{k_{0}}\right)\right)\right. \\
& \left.-\alpha_{0}\left(s+\frac{A_{0} T_{0}}{k_{0}}\right),\left(1-\alpha_{0}\right) y-x\right) .
\end{aligned}
$$

The Jacobi matrix of $G_{s}$ at $(0,0)$ has the form

$$
J G_{s}(0,0)=\left[\begin{array}{cc}
1 & \frac{\alpha_{0} k_{0}}{A_{0} T_{0}} s+\alpha_{0} \\
-1 & 1-\alpha_{0}
\end{array}\right],
$$

with the complex eigenvalues for sufficiently small $s$ of the form

$$
\lambda_{1,2}(s)=1-\frac{1}{2} \alpha_{0} \pm \frac{i}{2} \sqrt{4 \alpha_{0}\left(\frac{k_{0}}{A_{0} T_{0}} s+1\right)-\alpha_{0}^{2}}
$$

and modulus

$$
r(s)=\sqrt{1+\frac{\alpha_{0} k_{0}}{A_{0} T_{0}} s}
$$

Notice that $r(0)=1, r^{\prime}(0)=\alpha_{0} k_{0} / 2 A_{0} T_{0} \neq 0$, which means that condition (C.1) of Lemma 4 is satisfied. Now, we check that condition (C.2) (called no strong resonance) is satisfied. We have to prove that $e^{i l \theta_{0}} \neq 1$, where $l=1,2,3,4$ and $\theta_{0}$ is such that $\lambda_{1,2}(0)=r(0) e^{ \pm i \theta_{0}}$. We get for $\alpha_{0} \in(0,1)$

$$
\begin{aligned}
& 1-\frac{1}{2} \alpha_{0}+\frac{i}{2} \sqrt{4 \alpha_{0}-\alpha_{0}^{2}}=e^{i \theta_{0}} \neq 1, \\
& 1+\alpha_{0}\left(\frac{1}{2} \alpha_{0}-2\right)+i\left(1-\frac{1}{2} \alpha_{0}\right) \sqrt{4 \alpha_{0}-\alpha_{0}^{2}}=e^{2 i \theta_{0}} \\
& \quad \neq 1, \\
& 1-\frac{1}{2} \alpha_{0}\left(\alpha_{0}-3\right)^{2}+\frac{i}{2}\left(\alpha_{0}^{2}-4 \alpha_{0}+3\right) \sqrt{4 \alpha_{0}-\alpha_{0}^{2}} \\
& \quad=e^{3 i \theta_{0}} \neq 1, \\
& 1+\frac{1}{2} \alpha_{0}\left(\alpha_{0}^{3}-8 \alpha_{0}^{2}+20 \alpha_{0}-16\right) \\
& \quad+i\left(-\frac{1}{2} \alpha_{0}^{3}+3 \alpha_{0}^{2}-5 \alpha_{0}+2\right) \sqrt{4 \alpha_{0}-\alpha_{0}^{2}}=e^{4 i \theta_{0}} \\
& \quad \neq 1,
\end{aligned}
$$

because $2,2 \pm \sqrt{2}$ are solutions to equation $-(1 / 2) \alpha_{0}^{3}+3 \alpha_{0}^{2}$ $-5 \alpha_{0}+2=0$. Fulfilling conditions (C.1) and (C.2) from Lemma 4 means that nondegeneracy conditions are satisfied. To get the existence of Neimark-Sacker bifurcation, we have to check the last condition (C.3) of the form $a(0)=$ $\operatorname{Re}\left[e^{i \theta 0} c(0)\right] \neq 0$, named the first Lyapunov coefficient, given in Lemma 5. If $a(0)<0$, we get the supercritical NeimarkSacker bifurcation, and if $a(0)>0$ we get the subcritical Neimark-Sacker bifurcation. Due to complicated conditions for any parameters $A_{0}, T_{0}>0, \alpha_{0} \in(0,1)$, and $k_{0}>1$, we check the sign of the first Lyapunov coefficient only for specific $A_{0}, T_{0}, \alpha_{0}$, and $k_{0}$ in Section 3.

Remark 7. In the analogous way, we can prove the existence of the Neimark-Sacker one-parameter bifurcation of the planar system for any other parameters $A, T, k$, and $\alpha$ with the 


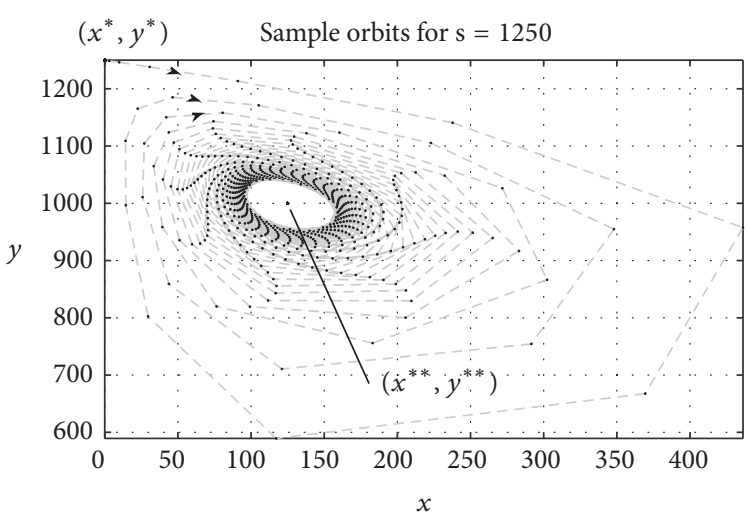

(a)

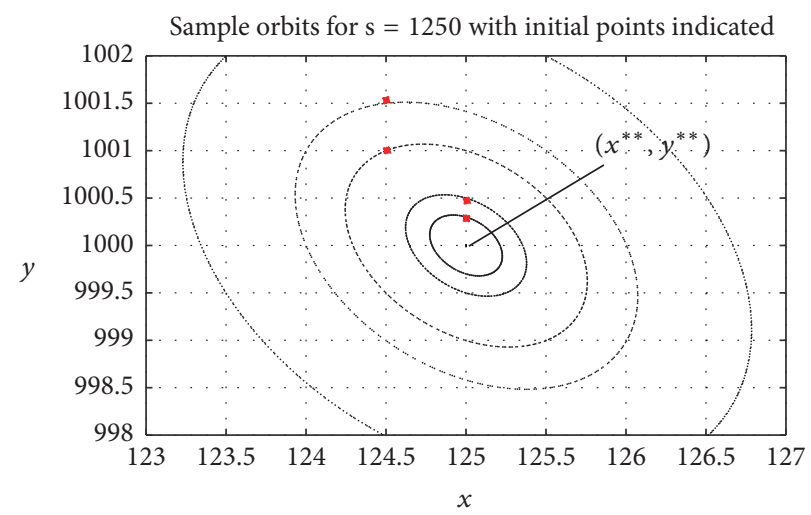

(c)

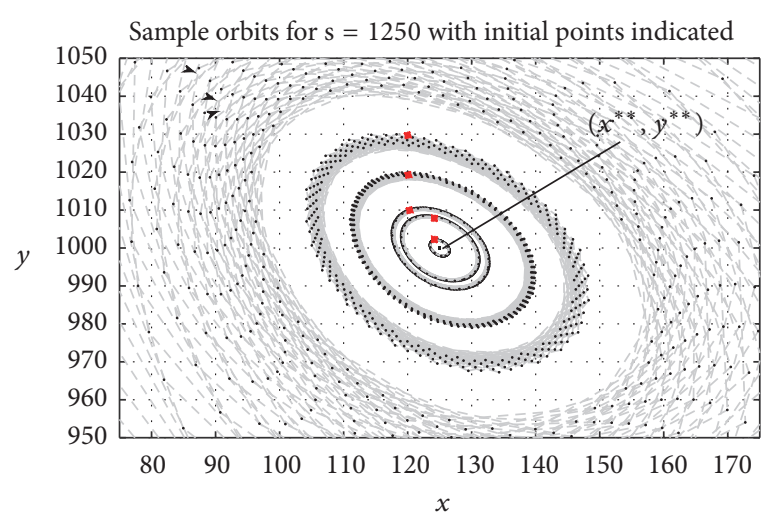

(b)

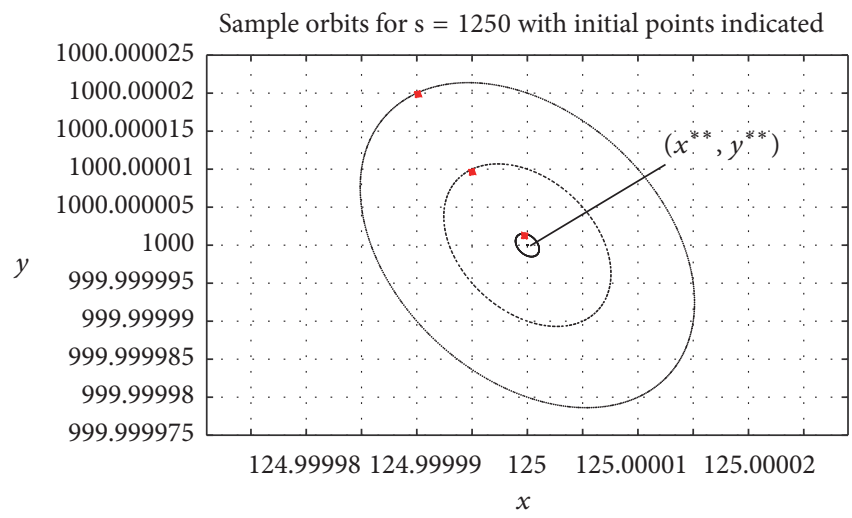

(d)

FIgURE 5: Dynamics of the case $s=(1+A / k) T$ with increasing zoom on the equilibrium point $\left(x^{* *}, y^{* *}\right)$.

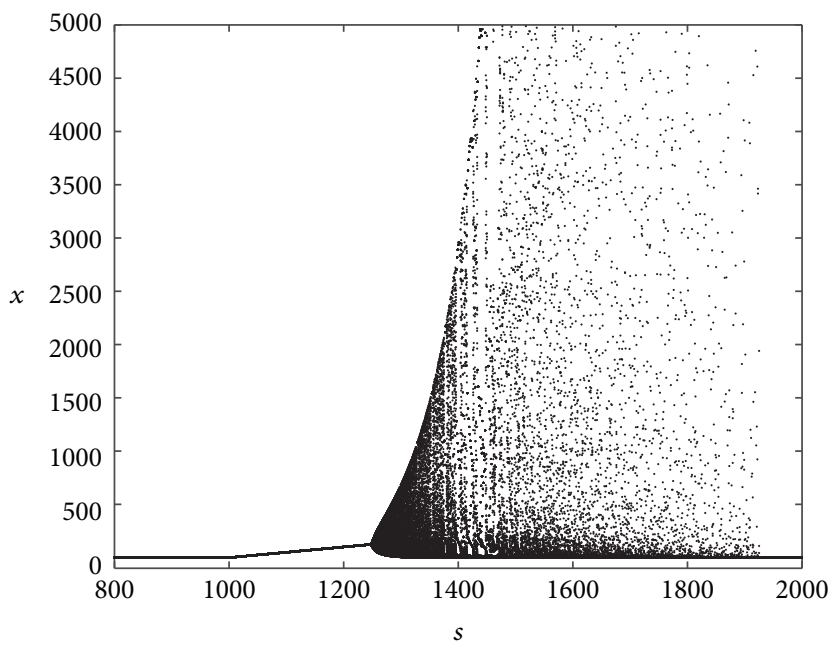

Figure 6: Diagram of bifurcation of the equilibrium points with respect to $x$, for the parameter $s$ varying from 800 to $2000, x_{1}=0.1, y_{1}=s$ for $A=1, T=1000, \alpha=0.5$, and $k=4$.

bifurcation parameter which fulfills the equality $s=(1+$ $A / k) T$.

\section{Numerical Simulations}

In this section, by using numeral simulation, we give the bifurcation diagrams and phase portraits of system (6) to confirm the previous analytical results and show new interesting complex dynamical behaviours emerging for system (6).

Now, let us give illustration to the cases provided in Table 1. Graphs in Figures 1-4 depict behaviour of (6) depending on the parameter $s$. The other parameters remain unchanged at $A=1, T=1000$, and $\alpha=0.5$, and, for $\mathrm{Cl}$, 


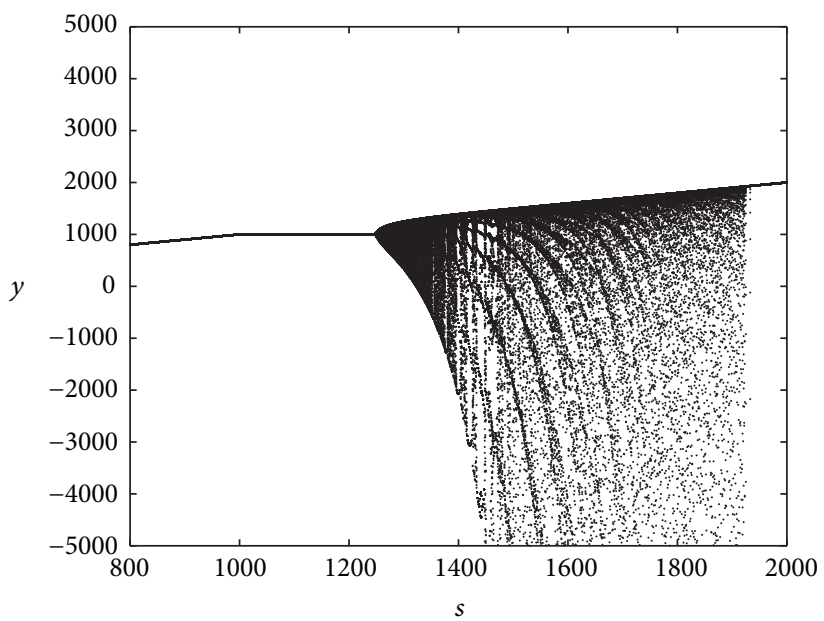

Figure 7: Diagram of bifurcation of the equilibrium points with respect to $y$, for the parameter $s$ varying from 800 to $2000, x_{1}=0.1, y_{1}=s$ for $A=1, T=1000, \alpha=0.5$, and $k=4$.

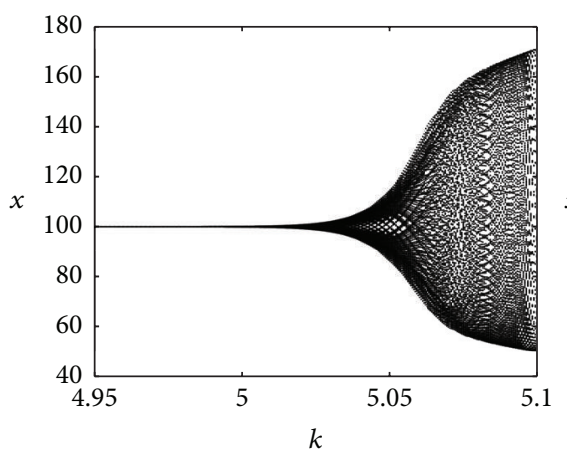

$k=4.95$ to 5.1

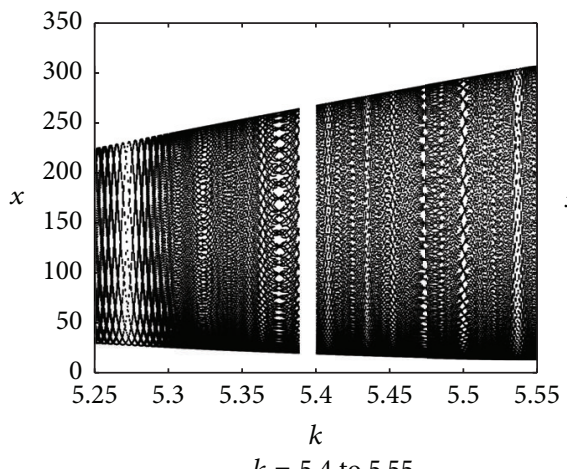

$k=5.4$ to 5.55

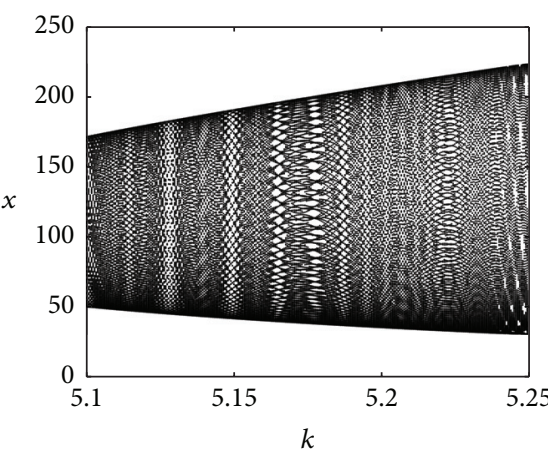

$k=5.1$ to 5.25

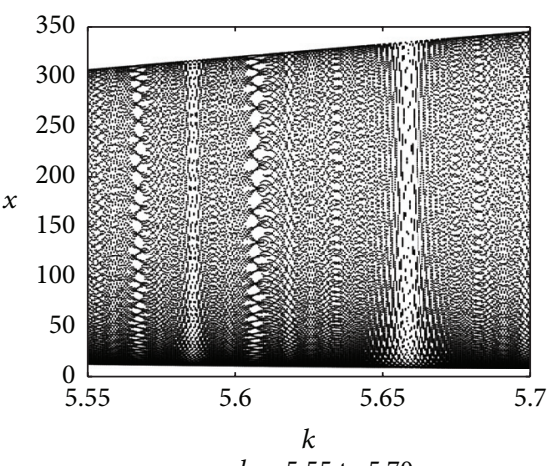

$k=5.55$ to 5.70

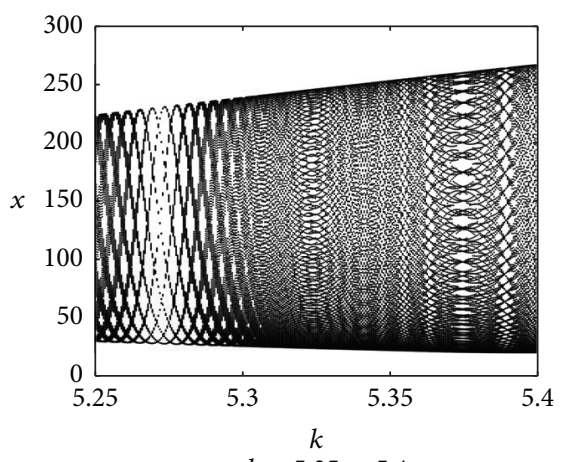

$k=5.25$ to 5.4

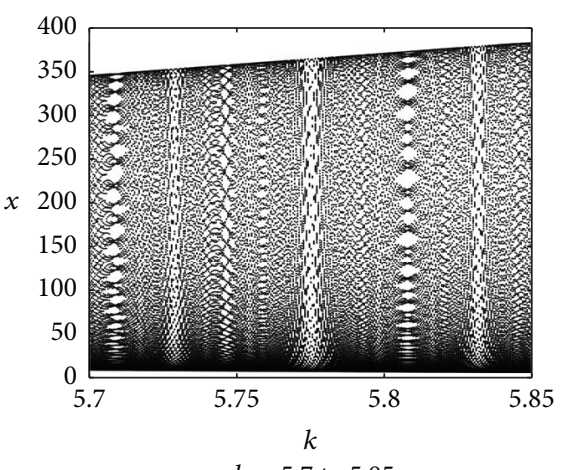

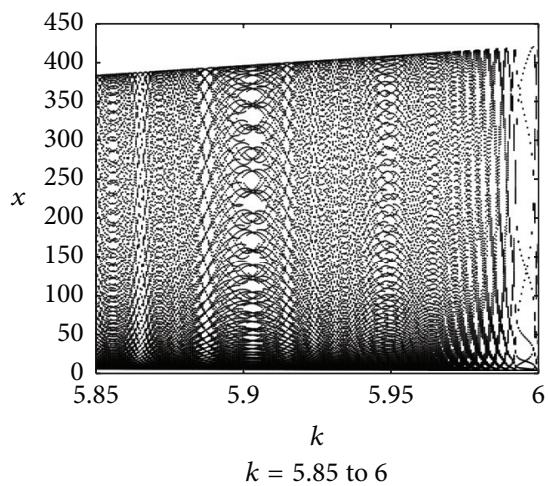

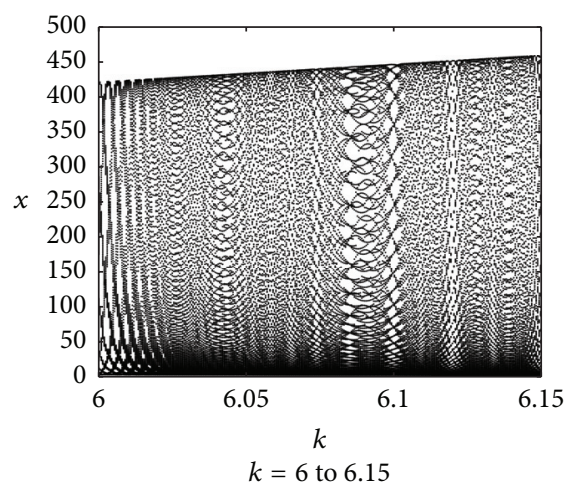

FIGURE 8: Diagrams of bifurcation of the equilibrium points with respect to $x$ for the parameter $k$ varying from 4.95 to $6.15, x_{1}=100.1$, $y_{1}=1000$ for $A=1, T=1000, \alpha=0.5$, and $s=1200$. 

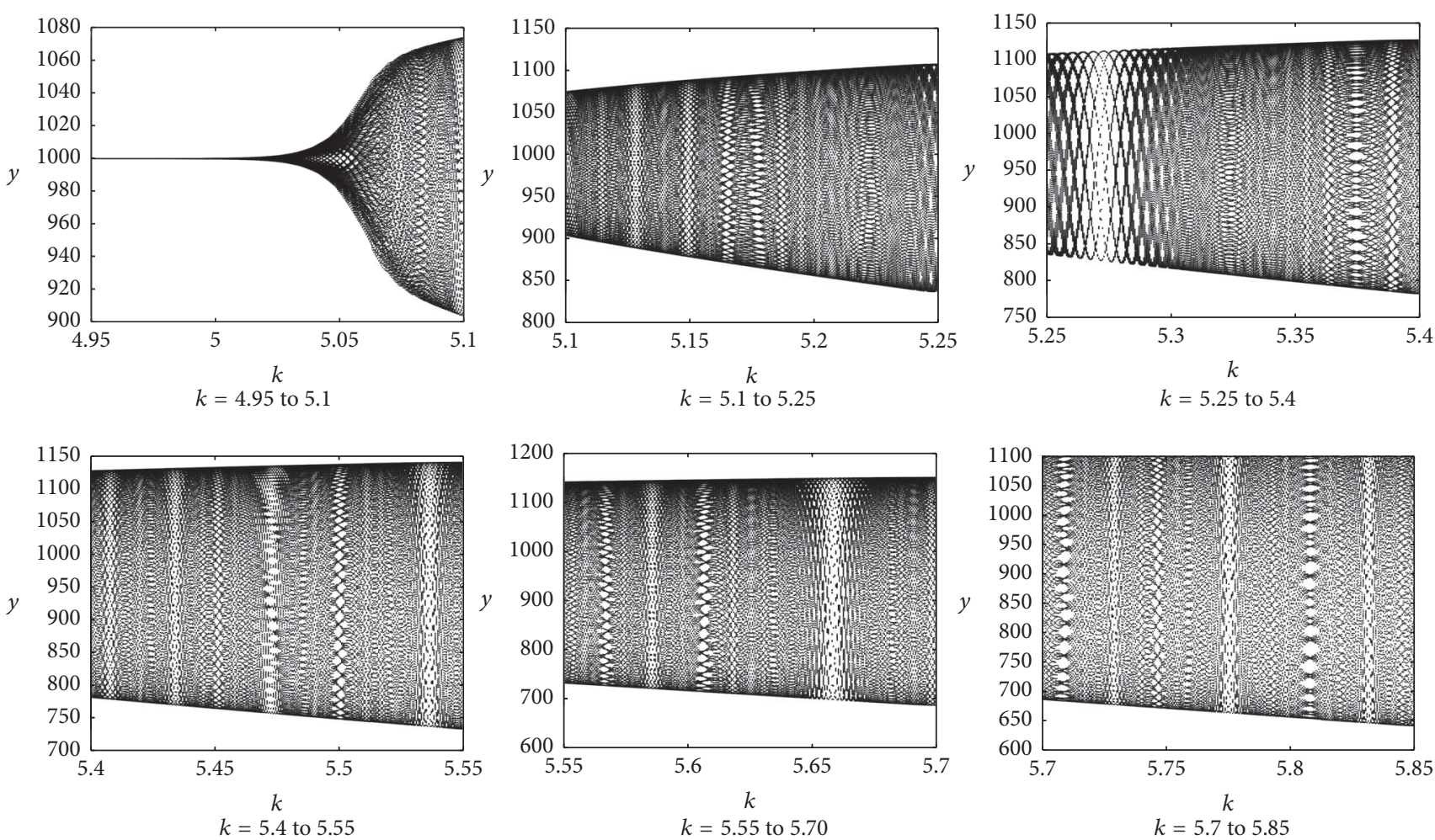

FIGURE 9: Diagrams of bifurcation of the equilibrium points with respect to $y$ for the parameter $k$ varying from 4.95 to $5.85, x_{1}=100.1$, $y_{1}=1000$ for $A=1, T=1000, \alpha=0.5$, and $s=1200$.

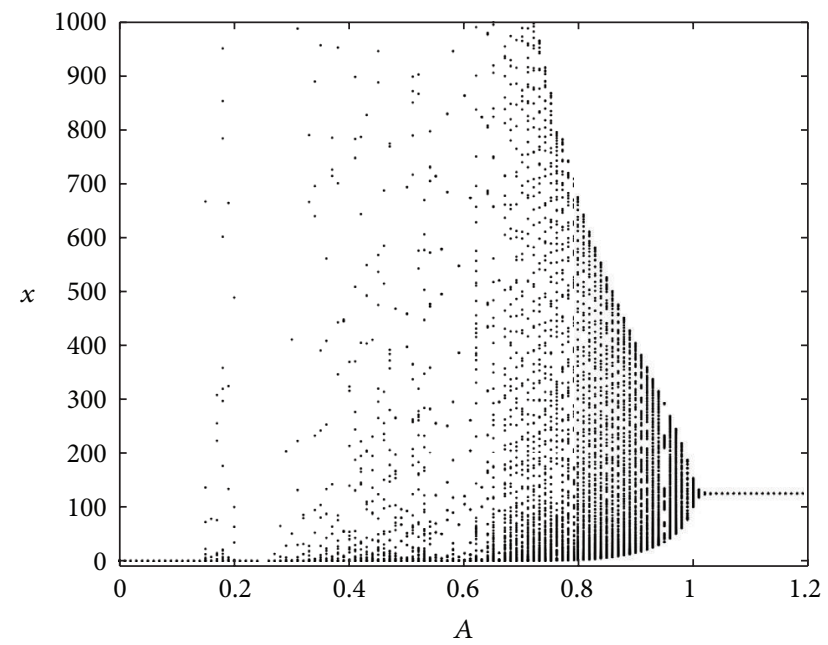

(a)

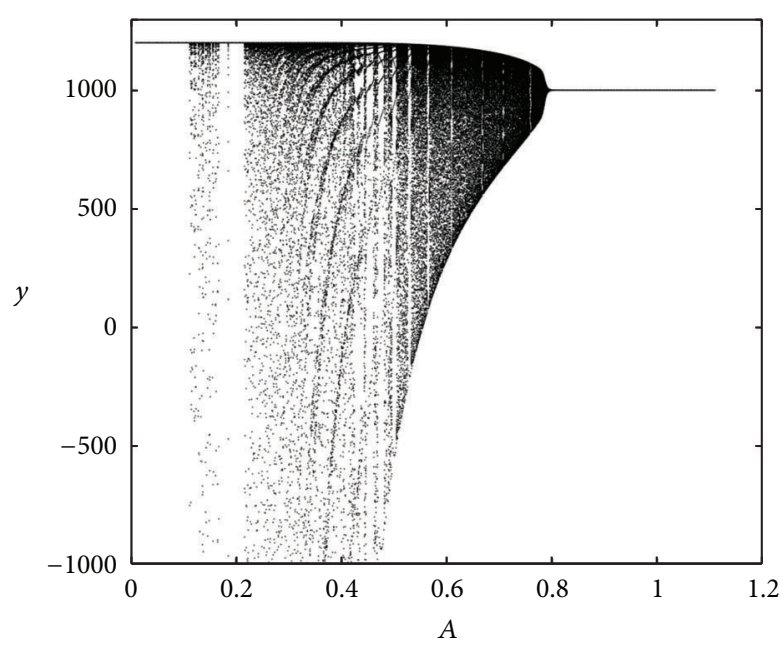

(b)

FIGURE 10: Diagrams of bifurcation of the equilibrium points with respect to $x$ (a) and $y$ (b), for the parameter $A$ varying from 0 to 1.2 , $x_{1}=0.1, y_{1}=s$ for $s=1250, T=1000, \alpha=0.5$, and $k=4$.

$\mathrm{C} 2$, C4, and $\mathrm{C} 5, k=4$; for (C3), $k=0.5$. The graphs clearly confirm the analytical results.

Interesting behaviour can be observed at border point $s=T$ depicted in Figures 1-2 in Graphs (C2a-b). At the first glance, in Graph (C2a), one can assume stability of the equilibrium point $(0, s)$, which would be a contradiction of the analytical result. However, looking from a closer perspective in Graph (C2b) one can see that the orbits leave the surroundings of the equilibrium point, herewith confirming the instability proved with analytical methods.

Let us have again a look at the border case of $s=(1+$ $A / k) T$ depicted in Figure 5. In each of the graphs, the same parameters are applied in order to observe the behaviour of the system for varying initial conditions of $x$ and $y(A=1$, $T=1000, \alpha=0.5, s=1250$, and $k=4$ ). Each time, we set the initial conditions closer and closer to the equilibrium 


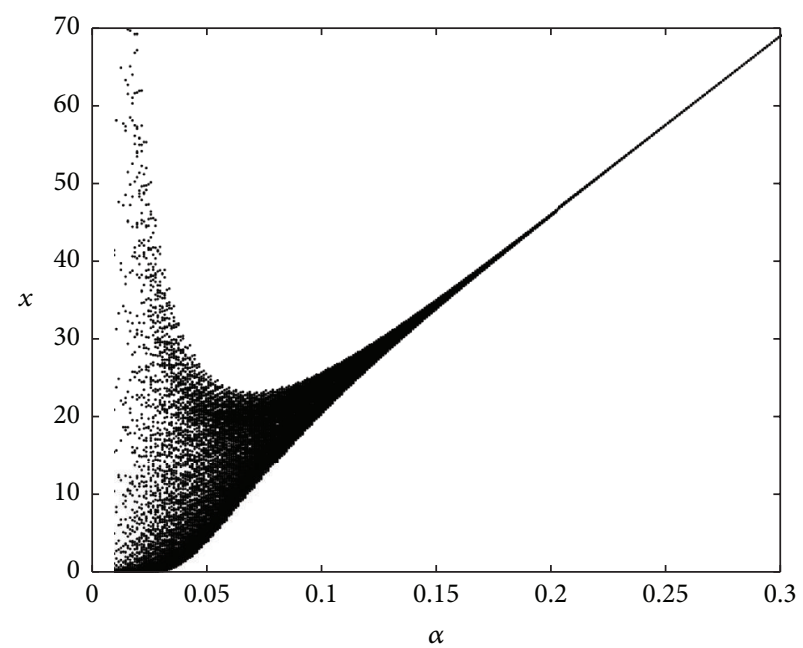

(a)

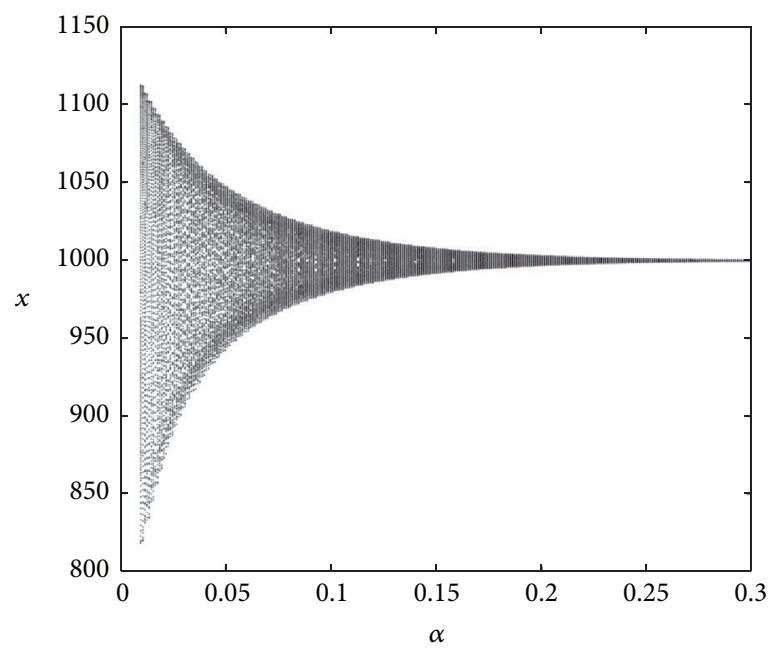

(b)

FIGURE 11: Diagram of bifurcation of the equilibrium points with respect to $x$ (a) and $y$ (b), for the parameter $\alpha$ varying from 0 to 0.3 , $x_{1}=100.1, y_{1}=s=1230$ for $A=1, T=1000$, and $k=4$.

point $\left(x^{* *}, y^{* *}\right)$. Appearance of this kind of circle suggests supercritical bifurcation of Neimark-Sacker type. Indeed, negative sign of the first Lyapunov coefficient $a(0)$ obtained numerically confirms that suggestion.

\section{Economical Interpretation}

System (6) depends on five parameters: $A, \alpha, T, k$, and $s$. Each of the parameters carries the economical meaning described in Section 1. In a business practice, the parameters $T$ and $s$ are set in advance. Nonetheless, as it is shown in Table 1, changes of parameter $s$ cause the change of behaviour of the equilibrium points. Therefore, we present bifurcation diagrams of $x$ and $y$ as $s$ changes in Figures 6 and 7 .

Parameter $k$, which represents price elasticity, is defined depending on the types of a product and a market and often is determined experimentally and must be adjusted frequently. Therefore, it is interesting to analyse behaviour of system (6) at varying values of this parameter. For that purpose, let us illustrate changes of the dynamics of the equilibrium points in the bifurcation diagrams (see Figures 8 and 9). Model (6) includes steering mechanism, which allows the user to influence the behaviour of the system. With the parameter $\alpha$, one can decide on the share of influence between the forecast and actuals of sales. The less $\alpha$ is, the greater the impact of actuals is, and vice versa. Figure 11 presents bifurcation diagram for changing parameter $\alpha$. Similarly, in Figure 10, we present bifurcation for varying $A$. $A$ is a parameter to control the influence of the discrepancy between the actual stock and the target one $T$.

Business Example. Let us consider a product $H_{R}$ that follows $\operatorname{demand}(D)$-price $(p)$ dependence $D_{2} / D_{1}=\left(p_{2} / p_{1}\right)^{-k}$ with price elasticity $k=4$. The target $T=1000$ is set on stock volume and assumed demand at least $z_{1}=50$. Actual demand is insufficient $x_{1}=1$, and hence the actual stock exceeds the target $y_{1}=1100>T$. Smoothing coefficient $\alpha=0.5$, which means calculation of the amount to be delivered takes into account actuals and forecast with equal wages. Parameter $A=$ 1 . One can easily calculate $s=y_{1}+z_{1} / \alpha=1200$. From Table 1 , it is observed that the product satisfies condition (C4), and hence the system has two equilibrium points $(0,1200)$ and $(100,1000)$, unstable and stable, respectively.

\section{Additional Points}

Numerical analysis and graph plotting have been performed using Matcontm (according to [27]) and Matlab R2016a. The bifurcation diagrams depict 200 iterations starting from iteration $n=801$.

\section{Competing Interests}

The authors declare that there are no competing interests regarding the publication of this paper.

\section{References}

[1] H. M. Wagner and T. M. Whitin, "Dynamic version of the economic lot size model," Management Science, vol. 5, no. 1, pp. 8996, 1958.

[2] R. G. Brown, Statistical Forecasting for Inventory Control, McGraw-Hill Book Co, New York, NY, USA, 1959.

[3] C. C. Holt, F. Modigliani, J. F. Muth, and H. A. Simon, Planning Production, Inventories, and Work Forces, Prentice-Hall, 1960.

[4] X. Qi, J. F. Bard, and G. Yu, "Supply chain coordination with demand disruptions," Omega, vol. 32, no. 4, pp. 301-312, 2004.

[5] S. R. T. Kumara, P. Ranjan, A. Surana, and V. Narayanan, "Decision making in logistics: a chaos theory based analysis," CIRP Annals-Manufacturing Technology, vol. 52, no. 1, pp. 381384, 2003.

[6] J. Ma and Y. Feng, "The study of the chaotic behavior in retailer's demand model," Discrete Dynamics in Nature and Society, vol. 2008, Article ID 792031, 12 pages, 2008. 
[7] X. Chen and P. Hu, "Joint pricing and inventory management with deterministic demand and costly price adjustment," Operations Research Letters, vol. 40, no. 5, pp. 385-389, 2012.

[8] S. K. Mondal, J. K. Dey, and M. Maiti, "A single period inventory model of a deteriorating item sold from two shops with shortage via genetic algorithm," Yugoslav Journal of Operations Research, vol. 17, no. 1, pp. 75-94, 2007.

[9] L. Nowakowska, "Dynamic discrete model for electricity price forecasting," in Proceedings of the 5th International Youth Conference on Energy (IYCE '15), Pisa, Italy, May 2015.

[10] P. Hachuła and E. Schmeidel, "Stability analysis of demandinventory model in a certain business case," in Proceedings of the 11th European Scientific Conference (TRANSCOM '15), University of Zilina, Žilina, Slovakia, June 2015.

[11] M. Nockowska-Rosiak, P. Hachuła, and E. Schmeidel, "Stability of equilibrium points of demand-inventory model with stockdependent demand," Journal of Difference Equations and Applications, vol. 22, no. 10, pp. 1490-1500, 2016.

[12] E. S. Gardner Jr., "Exponential smoothing: the state of the artpart II," International Journal of Forecasting, vol. 22, no. 4, pp. 637-666, 2006.

[13] H. R. Varian, Intermediate Microeconomics. A Modern Approach, W. W. Norton \& Company, New York, NY, USA, 8th edition, 2010.

[14] M. Migda, E. Schmeidel, and M. Zdanowicz, "Existence of nonoscillatory solutions for system of neutral difference equations," Applicable Analysis and Discrete Mathematics, vol. 9, no. 2, pp. 271-284, 2015.

[15] E. Thandapani, R. Karunakaran, and I. M. Arockiasamy, "Bounded nonoscillatory solutions of neutral type difference systems," Electronic Journal of Qualitative Theory of Differential Equations, p. 25, 2009.

[16] E. Thandapani and B. Ponnammal, "Oscillatory properties of solutions of three-dimensional difference systems," Mathematical and Computer Modelling, vol. 42, no. 5-6, pp. 641-650, 2005.

[17] E. Thandapani, M. Vijaya, and T. Li, "On the oscillation of third order half-linear neutral type difference equations," Electronic Journal of Qualitative Theory of Differential Equations, vol. 76, pp. 1-13, 2011.

[18] M. F. Aktaş, A. Tiryaki, and A. Zafer, "Oscillation of third-order nonlinear delay difference equations," Turkish Journal of Mathematics, vol. 36, no. 3, pp. 422-436, 2012.

[19] A. Andruch-Sobiło and M. Migda, "Bounded solutions of third order nonlinear difference equations," The Rocky Mountain Journal of Mathematics, vol. 36, no. 1, pp. 23-34, 2006.

[20] Z. Dolá and A. Kobza, "On third-order linear difference equations involving quasi-differences," Advances in Difference Equations, vol. 2006, Article ID 065652, 2006.

[21] Z. Liu, M. Jia, S. M. Kang, and Y. C. Kwun, "Bounded positive solutions for a third order discrete equation," Abstract and Applied Analysis, vol. 2012, Article ID 237036, 12 pages, 2012.

[22] J. Popenda and E. Schmeidel, "Nonoscillatory solutions of third order difference equations," Portugaliae Mathematica, vol. 49, no. 2, pp. 233-239, 1992.

[23] E. Schmeidel, "Oscillatory and asymptotically zero solutions of third order difference equations with quasidifferences," Opuscula Mathematica, vol. 26, no. 2, pp. 361-369, 2006.

[24] R. P. Agarwal, M. Bohner, S. R. Grace, and D. O’Regan, Discrete Oscillation Theory, Hindawi Publishing Corporation, New York, NY, USA, 2005.
[25] P. E. Kloeden and M. Rasmussen, Nonautonomous Dynamical Systems, vol. 176 of Mathematical Surveys and Monographs, American Mathematical Society, Providence, RI, USA, 2011.

[26] Y. A. Kuznetsov, Elements of Applied Bifurcation Theory, vol. 112, Springer, New York, NY, USA, 2nd edition, 1998.

[27] W. Govaerts, Yu. A. Kuznetsov, R. Khoshsiar Ghaziani, and H. G. E. Meijer, Cl MatContM: A Toolbox for Continuation and Bifurcation of Cycles of Maps, Universiteit Gent and Utrecht University, 2008. 


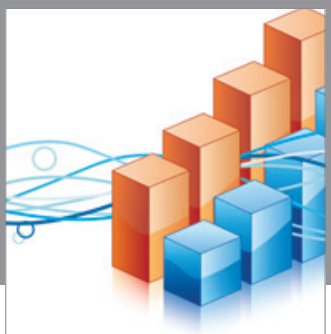

Advances in

Operations Research

vatem alat4

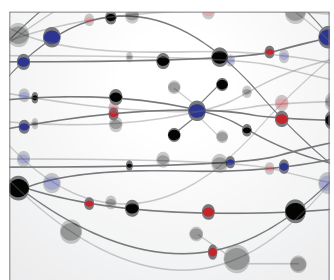

\section{The Scientific} World Journal
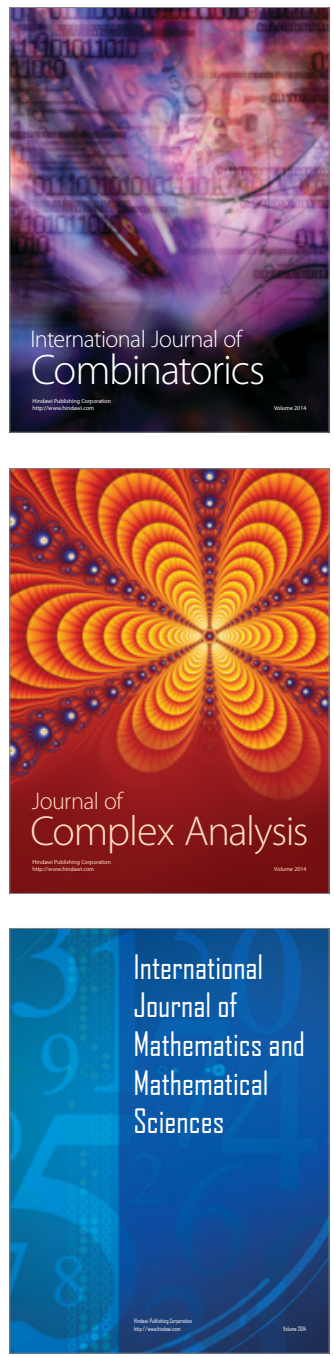
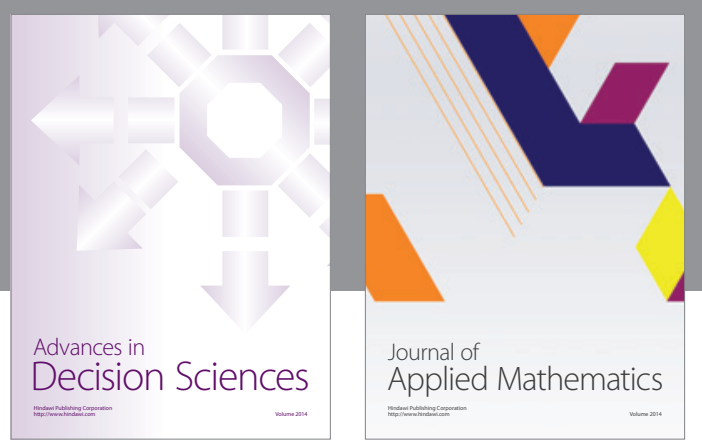

Algebra

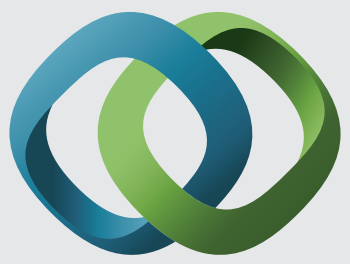

\section{Hindawi}

Submit your manuscripts at

https://www.hindawi.com
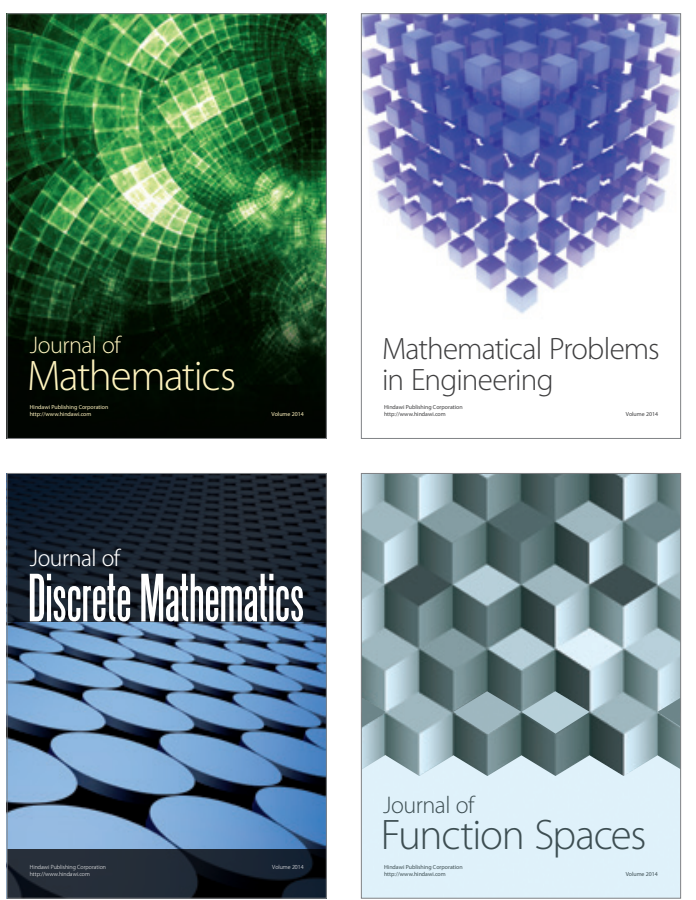

Mathematical Problems in Engineering
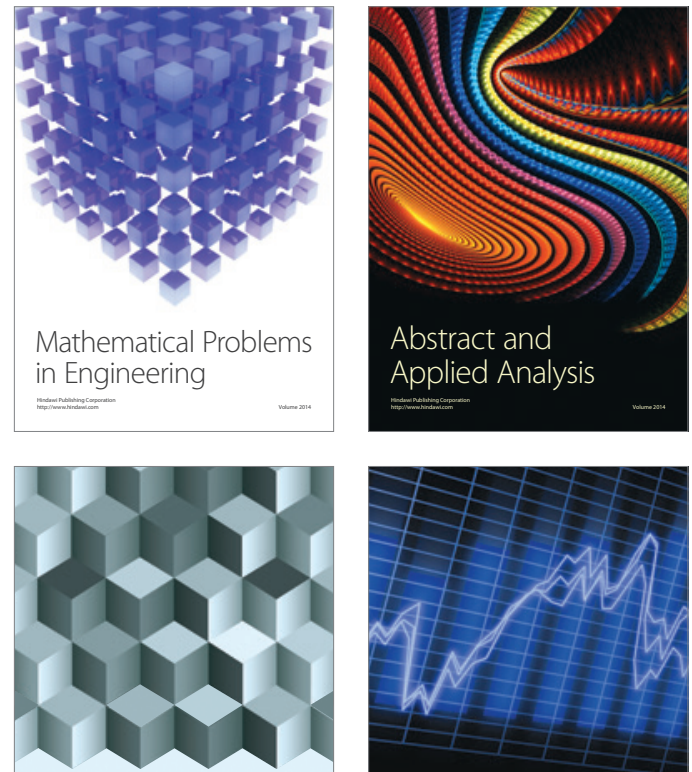

Journal of

Function Spaces

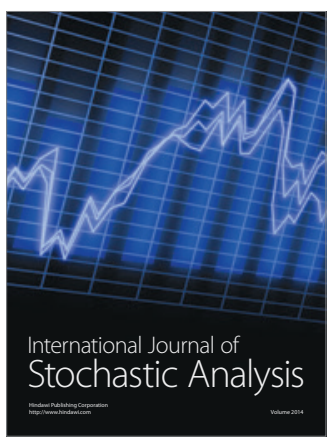

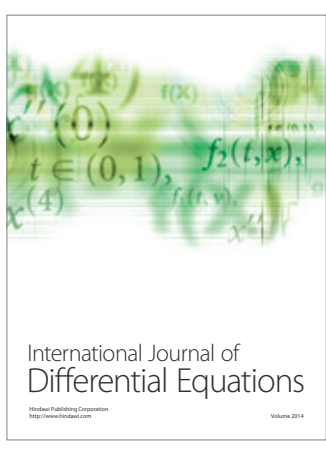
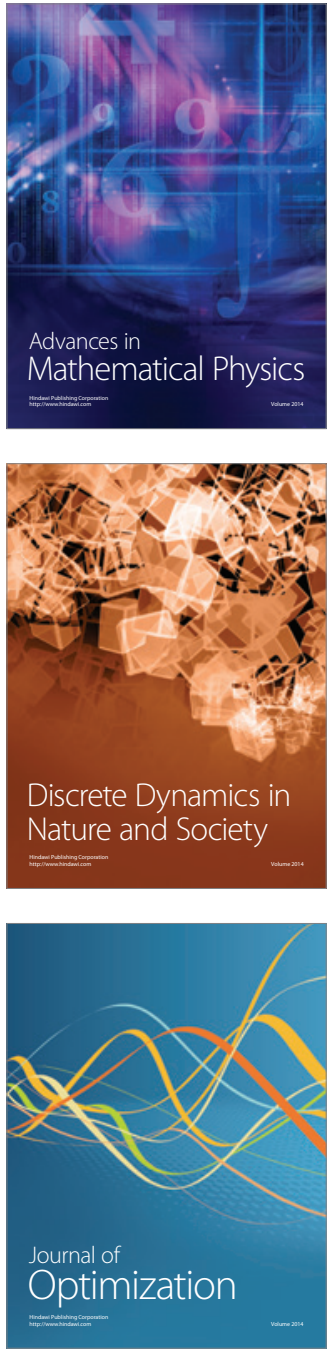